\title{
An Analytical Framework of a Deployment Strategy for Cloud Computing Services: A Case Study of Academic Websites
}

\author{
Chi-Hua Chen, ${ }^{1,2}$ Hui-Fei Lin, ${ }^{3}$ Hsu-Chia Chang, ${ }^{1}$ Ping-Hsien Ho, ${ }^{1}$ and Chi-Chun Lo ${ }^{1}$ \\ ${ }^{1}$ Institute of Information Management, National Chiao Tung University, 1001 University Road, Hsinchu 300, Taiwan \\ ${ }^{2}$ Telecommunication Laboratories, Chunghwa Telecom Co. Ltd., No. 12, Lane 551, Min-Tsu Road Sec. 5, \\ Yang-Mei, Taoyuan 326, Taiwan \\ ${ }^{3}$ Department of Communication and Technology, National Chiao Tung University, 1001 University Road, Hsinchu 300, Taiwan
}

Correspondence should be addressed to Chi-Hua Chen; chihua0826@gmail.com

Received 9 April 2013; Revised 14 July 2013; Accepted 1 August 2013

Academic Editor: Yi-Chung Hu

Copyright (C) 2013 Chi-Hua Chen et al. This is an open access article distributed under the Creative Commons Attribution License, which permits unrestricted use, distribution, and reproduction in any medium, provided the original work is properly cited.

\begin{abstract}
Cloud computing has become a popular topic for exploration in both academic and industrial research in recent years. In this paper, network behavior is analyzed to assess and compare the costs and risks associated with traditional local servers versus those associated with cloud computing to determine the appropriate deployment strategy. An analytic framework of a deployment strategy that involves two mathematical models and the analytical hierarchy process is proposed to analyze the costs and service level agreements of services involving using traditional local servers and platform as service platforms in the cloud. Two websites are used as test sites to analyze the costs and risks of deploying services in Google App Engine (GAE) (1) the website of Information and Finance of Management (IFM) at the National Chiao Tung University (NCTU) and (2) the NCTU website. If the examined websites were deployed in GAE, NCTU would save over $83.34 \%$ of the costs associated with using a traditional local server with low risk. Therefore, both the IFM and NCTU websites can be served appropriately in the cloud. Based on this strategy, a suggestion is proposed for managers and professionals.
\end{abstract}

\section{Introduction}

Cloud computing can provide high scalability and powerful computing capabilities, enable rapid deployment, and reduce costs for businesses [1, 2]; therefore, cloud computing is increasingly relevant. The current cloud computing service (CCS) vendors (e.g., Google, Microsoft, Salesforce.com, and Amazon.com) provide CCS requesters with three different architectures for their service deployment: infrastructure as a service (IaaS), platform as a service ( $\mathrm{PaaS}$ ), and software as a service (SaaS) [3]. IaaS enables substituting a hardware infrastructure with a platform virtualization environment (e.g., a virtual private server) to offer businesses fully outsourced service [4]. PaaS provides a higher level of abstraction and can support the integration of design, development, deployment, testing, and hosting for CCS requesters [5]. SaaS provides business application software through the Internet.

In recent years, numerous businesses have transferred their services PaaS platforms (e.g., Google App Engine (GAE),
Amazon Web Service (AWS), and Microsoft Azure), especially onto GAE, which can provide automatic and optimal resource allocation with scaling and load-balancing techniques. GAE also provides supports dynamic web service with full support for common web technologies and reliable storage with queries, sorting, and transactions. In addition, applications run in a secure environment with limited access to the underlying operating system in the sandbox [6]. Several factors affect CCS deployment strategies in the PaaS platform. Cloud computing providers and other parties such as service consumers and companies sign service level agreements (SLAs) to order their desired level of cloud computing service from a provider. Armbrust et al. [7] showed that the cost and the service blocking rate (SBR) are crucial factors in determining the deployment strategy. Although cloud computing can support utility computing and pay-per-use for on-demand scalability, the CCS requester is charged a high unit cost by CCS vendors. Furthermore, the available rate of CCS in accordance with SBR is considered for SLAs. 
TABLE 1: The competitive matrix diagrams of CCS vendors [8].

\begin{tabular}{lccc}
\hline Provider & Google App Engine & Microsoft Windows Azure & Amazon Web Services \\
\hline IaaS & No & No & Yes \\
PaaS & Yes (Python, Java) & Yes (.Note, Java, Ruby, Python, PHP) & No \\
Compute billing model & Pay-per-use & Pay-per-use & Pay-per-use \\
Storage billing model & Pay-per-use & Pay-per-use & Pay-per-use \\
Relational database service & Yes (MySQL based) & Yes (SQL Server) & No \\
\hline
\end{tabular}

TABLE 2: Request free and billing-enabled quota [6].

\begin{tabular}{|c|c|c|c|c|}
\hline \multirow{2}{*}{ Resource } & \multicolumn{2}{|c|}{ Free default quota } & \multicolumn{2}{|c|}{ Billing-enabled default quota } \\
\hline & Daily limit & Maximum rate & Daily limit & Maximum rate \\
\hline Requests (includes HTTPS) & $43,200,000$ requests & 45,200 requests/minute & $43,200,000$ requests & $\begin{array}{l}45,200 \\
\text { requests/minute }\end{array}$ \\
\hline $\begin{array}{l}\text { Outgoing bandwidth (billable, } \\
\text { includes HTTPS) }\end{array}$ & $1 \mathrm{~GB}$ & $56 \mathrm{MB} /$ minute & $\begin{array}{l}1 \mathrm{~GB} \text { free; } 14,400 \mathrm{~GB} \\
\text { maximum }\end{array}$ & $10 \mathrm{~GB} /$ minute \\
\hline $\begin{array}{l}\text { Incoming bandwidth (billable, } \\
\text { includes HTTPS) }\end{array}$ & $1 \mathrm{~GB}$ & $56 \mathrm{MB} /$ minute & $\begin{array}{l}1 \mathrm{~GB} \text { free; } 14,400 \mathrm{~GB} \\
\text { maximum }\end{array}$ & $10 \mathrm{~GB} /$ minute \\
\hline CPU time (billable) & 6.5 CPU hours & $15 \mathrm{CPU}$ minutes/minute & $\begin{array}{c}\text { 6.5 CPU hours free; } \\
\text { 1,729 CPU hours } \\
\text { maximum }\end{array}$ & $\begin{array}{l}72 \mathrm{CPU} \\
\text { minutes/minute }\end{array}$ \\
\hline
\end{tabular}

Based on the relationship between demand and capacity, an analytic framework of a deployment strategy that combines the analytical hierarchy process (AHP) to analyze the cost and an SLA based on the SBR of the CCS is proposed. Two websites are used as examples (1) the Information and Finance of Management (IFM) page on the National Chiao Tung University (NCTU) website and (2) the NCTU website. In this paper, network behaviors and the deployment factors of PaaS, which include the cost and SLA for CCS deployment strategy making, are analyzed.

The remainder of the paper is organized as follows. Background knowledge and relevant technologies are presented in Section 2. In Section 3, an analytic framework of a deployment strategy is proposed to analyze the cost of services and SLA, and those services are compared between the PaaS platform and a traditional local server. In Section 4, the parameters of the model affecting the cost and SLA are examined. An analysis of network behavior is also included to assess the platforms that are suitable for CCS deployment. Actual data are gathered from the IFM and the log files of NCTU websites. Finally, conclusions and recommendations for future work are offered in Section 5.

\section{Literature Review}

Necessary research background and relevant technologies include (1) CCS, (2) network behaviors, and (3) deployment strategy.

2.1. Cloud Computing Service. The current CCS venders (e.g., Google, Microsoft, and Amazon) provide different architectures to CCS requesters for their service deployment.

Table 1 shows the characteristics of major computing vendors including the types of services providing and billing models that they offer [8]. First, most CCS vendors provide a PaaS platform for users to deploy their services and use various programming languages. Second, all vendors support a pay-per-use billing model. Because the website is suitable to be deployed on PaaS platform and the deployment cost of GAE is cheaper than that of Microsoft Windows Azure, a deployment strategy using GAE is discussed and analyzed in this study.

The costs of the GAE PaaS platform include requests, outgoing bandwidth, incoming bandwidth, and CPU time, as shown in Table 2 . The default quota is divided into free quota and billing-enabled quota. For example, when the outgoing bandwidth is less than $1 \mathrm{~GB}$, GAE does not charge a fee. However, when the bandwidth is higher than this amount, GAE charges customers, and the fees are based on the amount exceeding the free default quota.

Cloud computing involves scalability and availability. The SBR of the PaaS platform may occur because of the limits of the billing-enabled default quota. These daily limits or the maximal rate per minute affects the risk of building services on a cloud. This study explores the costs of CCSs and the billing-enabled default quota to derive SBR models for SLAs.

Table 3 shows the cost for computing resources. In Section 4 , these costs are adopted to determine the deployment cost for a CCS.

2.2. Network Behaviors. Certain network behaviors have been defined, these include network path, interarrival patterns, and transmission control protocol (TCP) behavior patterns. Mogul used interarrival patterns which include packet arrivals, request arrivals, and per-client request arrivals to analyze HTTP server logs. Then the interarrival distribution was obtained for several categories of events [9]. Inter-arrival patterns are suitable to be used in generating a probability density function for the analyzing of a web server. Therefore, 
TABLE 3: Billable quota unit cost [6].

\begin{tabular}{lcc}
\hline Resource & Unit & Unit cost \\
\hline Outgoing bandwidth & Gigabytes & $\$ 0.12$ \\
Incoming bandwidth & Gigabytes & $\$ 0.10$ \\
CPU time & CPU hours & $\$ 0.10$ \\
Stored data & Gigabytes per month & $\$ 0.15$ \\
High replication storage & Gigabytes per month & $\$ 0.45$ \\
Recipients emailed & Recipients & $\$ 0.0001$ \\
Always on & N/A (daily) & $\$ 0.30$ \\
\hline
\end{tabular}

interarrival patterns are used to collect the server logs and obtain the interarrival distribution for analyzing the cost and SLA of a CCS.

2.3. Deployment Strategy. Few studies have addressed the deployment strategy for a CCS. Therefore, this section first presents previous studies concerning the deployment strategy for a CCS. Then AHP which is a popular multiple-attribute decision-making method is described and discussed for use in the deployment strategy.

2.3.1. Deployment Strategy for a Cloud Computing Service. Kang and Sim proposed a multicriteria cloud service search engine in which cloud ontology and the similarity reasoning method are considered to support matching algorithms by using three types of requirements. These requirements are (1) the functional requirement, (2) the technical requirement, and (3) the cost requirement [10]. Although their simulation results indicate that this engine can be used to search the adapter private cloud framework, the weight of each requirement is subjectively defined in the engine which is unsuitable for a public cloud.

Some researchers have used the Amazon cloud fee structure and a real-life astronomy application. They considered the tradeoffs of different execution and resource provisioning plans in the simulation. When used for long term application data archival, they also studied the tradeoffs in the context of the storage and communication fees of Amazon S3. Their results show that cost can be markedly reduced without a substantial impact on application performance by providing the suitable amount of storage and computing resources [11]. However, they only focused on the resource deployment of Amazon, and the differences in deployment between local servers and the cloud platform were not compared.

2.3.2. Analytical Hierarchy Process. The AHP, which was proposed by Saaty, is typically used to model multiple-attribute decision making in a hierarchical system [12]. Therefore, the AHP is used to analyze and calculate the weight of each factor for decision making. The main steps of the AHP are described as follows [13].

The evaluative criteria are defined and the hierarchical system is established in Step 1.

The comparative weight between the attributes of the decision elements is calculated to generate the reciprocal matrix in Step 2. For example, a set of $n$ compared attributes are denoted by $a_{1}, a_{2}, \ldots, a_{n}$ and the weights are defined as $w_{1}, w_{2}, \ldots, w_{n}$. They are shown in (1), and $w_{i}$ is defined as $\sum_{y=1}^{n}\left(a_{i y} / \sum_{x=1}^{n} a_{x y}\right) / n$. For consistency measurement, the consistency index (CI), which is defined in (2), was proposed by Saaty, who suggested that the value of the CI should not exceed 0.1 for a confident result $[13,14]$.

Consider

$$
\begin{aligned}
A & =\left[\begin{array}{ccccc}
a_{11} & \cdots & a_{1 j} & \cdots & a_{1 n} \\
\vdots & & \vdots & & \vdots \\
a_{i 1} & \cdots & a_{i j} & \cdots & a_{i n} \\
\vdots & & \vdots & & \vdots \\
a_{n 1} & \cdots & a_{n j} & \cdots & a_{n n}
\end{array}\right] \\
& \cong\left[\begin{array}{ccccc}
\frac{w_{1}}{w_{1}} & \cdots & \frac{w_{1}}{w_{j}} & \cdots & \frac{w_{1}}{w_{n}} \\
\vdots & & \vdots & & \vdots \\
\frac{w_{i}}{w_{1}} & \cdots & \frac{w_{i}}{w_{j}} & \cdots & \frac{w_{i}}{w_{n}} \\
\vdots & & \vdots & & \vdots \\
\frac{w_{n}}{w_{1}} & \cdots & \frac{w_{n}}{w_{j}} & \cdots & \frac{w_{n}}{w_{n}}
\end{array}\right]=W
\end{aligned}
$$

where $a_{i j}>0, a_{i j}=1 / a_{j i}$, and $w_{i}=\sum_{y=1}^{n}\left(a_{i y} / \sum_{x=1}^{n} a_{x y}\right) / n$.

Consider

$$
\mathrm{CI}=\frac{\lambda-n}{n-1}
$$

where $\lambda=\sum_{i=1}^{n}\left(\left(\sum_{j=1}^{n} w_{j} a_{i j}\right) / w_{i}\right) / n$.

The hierarchy weight at each level is calculated to determine the optimal strategy in Step 3.

\section{An Analytic Framework of the Deployment Strategy for a Cloud Computing Service}

This section proposes an analytic framework based on network behavior for the deployment strategy of a CCS (shown in Figure 1). First, this study uses the curve-fitting method [15] which was used to determine the probability distribution for interarrival patterns (Figure 1(a)). The queueing theory is then used to measure the considered criteria which include costs and the SLA in accordance with network behaviors (Figure 1(b)). Finally, this study uses these criteria, and the AHP to set up the hierarchy system for decision making (Figure 1(c)).

3.1. Network Behaviors for a Cloud Computing Service. Two network behaviors are considered in this study: they are the probability distribution of the request arrival process and service time. First, the request logs are collected from the web server to generate the probability distribution. Then the curve-fitting method is used based on the chi-square test to assume and verify whether the probability distribution fits the assumed probability density function (e.g., Poisson distribution and exponential distribution). 


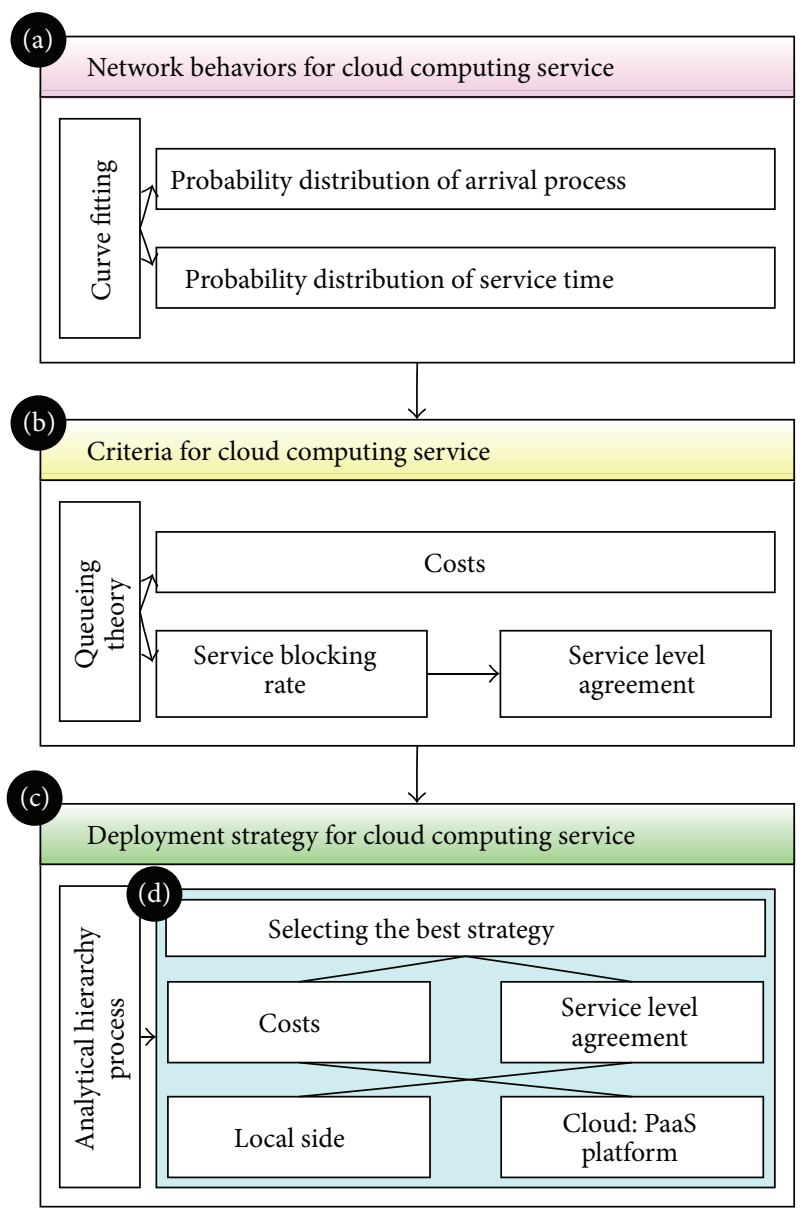

FIGURE 1: The proposed analytic framework of deployment strategy for CCS.

3.2. Criteria for a Cloud Computing Service. The proposed analytic framework of the deployment strategy includes two criteria. For the first criterion, the costs associated with the PaaS platform versus those associated with a traditional local server are calculated. For the second criterion, the SLA is measured based on the SBRs of the PaaS platform and a traditional local server.

3.2.1. Criterion 1: Analysis of Costs. The analysis of service costs for the PaaS platform versus those of a local server is divided into two parts, the first for the PaaS platform and the second for the local server.

(1) Local Side. The costs associated with a traditional local server include network costs $\left(C_{N}\right)$, electricity $\left(C_{E}\right)$, server depreciation expenses $\left(C_{\mathrm{SDE}}\right)$, and personnel costs $\left(C_{P}\right)$. Equation (3) is assumed to be the total cost $\left(C_{L}\right)$ of the traditional local server. Table 4 lists the parameters of (3).

Consider

$$
C_{L}=C_{N}+C_{E}+C_{\mathrm{SDE}}+C_{P}
$$

(2) Cloud: PaaS Platform. A probability density function $\operatorname{Pr}(k)$ is assumed in which the number of requests is equal to $k$ for a one-day period. The average amount of data per request
TABLE 4: Cost parameters for a traditional local server.

\begin{tabular}{lc}
\hline Parameter & Description \\
\hline$C_{L}$ & Total cost of a traditional local server \\
$C_{N}$ & Network costs \\
$C_{E}$ & Electricity costs \\
$C_{\mathrm{SDE}}$ & Server depreciation expenses \\
$C_{P}$ & Personnel costs \\
\hline
\end{tabular}

TABLE 5: Cost parameters for CCS.

\begin{tabular}{lc}
\hline Parameter & Description \\
\hline$k$ (request) & $\begin{array}{c}\text { Number of requests per day } \\
\operatorname{Pr}(k)\end{array}$ \\
$\xi$ & $\begin{array}{c}\text { Probability density function when the } \\
\text { number of requests is equal to } k\end{array}$ \\
$r$ & Average amount of data per request \\
$C_{G}$ & Maximum number of requests per day \\
$C_{C}$ & Cost per gigabyte \\
\hline
\end{tabular}

is defined as $\xi$, and the cost per gigabyte is defined as $C_{G}$. In the case of GAE, for example, there is a free daily throughput of $1 \mathrm{~GB}$. The CCS cost $\left(C_{C}\right)$ can be calculated using (4). Table 5 lists the parameters of (4).

Consider

$$
C_{C}=\left(\left\lceil\xi \times \sum_{k=0}^{r} k \times \operatorname{Pr}(k)\right\rceil-1\right) \times C_{G} .
$$

3.2.2. Criterion 2: Analysis of the Service Level Agreement. Because local servers have limited computing power compared to high-capacity cloud computing services, this section proposes a SLA model based on the SBR for comparing the traditional local server with the PaaS platform.

(1) Local Side. There is a ceiling on the number of users a local server can service simultaneously; thus, the SBR is assumed using (5) to represent the probability $\operatorname{Pr}_{l}(c)$ that the number of requests for service exceeds the maximal online capacity. The SLA is defined in (6). Table 6 lists the parameters of (5) and (6).

Consider

$$
\begin{aligned}
& B_{L}=\operatorname{Pr}_{l}(c), \\
& S_{L}=1-B_{L} .
\end{aligned}
$$

(2) Cloud: PaaS Platform. PaaS vendors typically limit the number of acceptable requests $(n)$ per minute or per day. Therefore, a probability density function $\operatorname{Pr}(k)$ is assumed in which the number of requests is equal to $k$ for a 1 minute or 1 day period; the SBR $\left(B_{C}\right)$ in the PaaS platform is defined in (7), and the SLA is defined in (8). Table 7 lists the parameters of (7) and (8). 
TABLE 6: SLA parameters for a traditional local server.

\begin{tabular}{lc}
\hline Parameter & Description \\
\hline$B_{L}, \operatorname{Pr}_{l}(c)$ & $\begin{array}{c}\text { Probability function when the maximum } \\
\text { service limit } c \text { is reached }\end{array}$ \\
$S_{L}$ & SLA of a traditional local server \\
\hline
\end{tabular}

TABLE 7: SLA parameters for CCS.

\begin{tabular}{|c|c|}
\hline Parameter & Description \\
\hline$B_{C}$ & Probability of the SBR on a PaaS platform \\
\hline$k$ & Number of requests per minute or day \\
\hline $\operatorname{Pr}(k)$ & $\begin{array}{l}\text { Probability density function when the } \\
\text { number of requests is equal to } k\end{array}$ \\
\hline$n$ & $\begin{array}{l}\text { The maximum allowable number of } \\
\text { accumulated requests per minute or day }\end{array}$ \\
\hline$S_{C}$ & SLA on a PaaS platform \\
\hline
\end{tabular}

Consider

$$
\begin{gathered}
B_{C}=\sum_{k=n+1}^{\infty} \operatorname{Pr}(k), \\
S_{C}=1-B_{C}=1-\sum_{k=n+1}^{\infty} \operatorname{Pr}(k) .
\end{gathered}
$$

3.3. The Deployment Strategy for a Cloud Computing Service. In this study, the AHP is used to analyze the comparative weight between the attributes of the decision elements in a hierarchical system (Figure 1(d)). The criteria include costs and the SLA, and the strategies include local side and the CCS. Therefore, six matrices can be calculated by using (1) as follows:

(1) matrices $A_{R}$ and $W_{R}$ (shown in (9)) for the weight of the criteria divided by the given cost weight $w_{c}$ and SLA weight $w_{s}$,

(2) matrices $A_{C}$ and $W_{C}$ (shown in (10)) for the weight of strategy based on costs in accordance with (3) and (4),

(3) matrices $A_{S}$ and $W_{S}$ (shown in (11)) for the weight of the strategy based on the SLA in accordance with (6) and (8).

One has

$$
A_{R}=\operatorname{Cost}\left[\begin{array}{cc}
1 & \frac{w_{C}}{w_{S}} \\
\frac{w_{S}}{w_{C}} & 1
\end{array}\right]=W_{R}
$$

where $w_{C}>0, w_{S}>0$.
One has

$$
\begin{aligned}
A_{C}= & \text { Local }\left[\begin{array}{cc}
\text { Local } & \text { Cloud } \\
1 & \text { Cloud }
\end{array} a_{C, L C}=\frac{C_{C}}{C_{L}}\right. \\
\cong & \text { Local }\left[\begin{array}{cc}
1 & \frac{w_{C, L}}{C_{C}} \\
\frac{w_{C, C}}{w_{C, C}} & 1
\end{array}\right]=W_{C},
\end{aligned}
$$

where $w_{C, L}=C_{C} /\left(C_{L}+C_{C}\right)$ and $w_{C, C}=C_{L} /\left(C_{L}+C_{C}\right)$.

One has

$$
\begin{aligned}
A_{S}= & \operatorname{Local}\left[\begin{array}{cc}
\text { Local } & \text { Cloud } \\
1 & a_{S, L C}=\frac{S_{L}}{S_{C}} \\
a_{S, C L}=\frac{S_{C}}{S_{L}} & 1
\end{array}\right] \\
& \text { Cloud } \\
& \text { Clocal }\left[\begin{array}{cc}
1 & \frac{w_{S, L}}{w_{S, C}} \\
\frac{w_{S, C}}{w_{S, L}} & 1
\end{array}\right]=W_{S},
\end{aligned}
$$

where $w_{S, L}=S_{L} /\left(S_{L}+S_{C}\right)$ and $w_{S, C}=S_{C} /\left(S_{L}+S_{C}\right)$.

For consistency measurement, (2) is used to test (9), (10), and (11). Because all CIs (shown in (12)) are equal to 0 , the results are valid.

Consider

$$
\text { CI for } A_{R}: \frac{\lambda-2}{2-1}=\frac{2-2}{1}=0,
$$

where $\lambda=\frac{\left(w_{C}+w_{S} \times\left(w_{C} / w_{S}\right)\right) / w_{C}}{2}$

$$
\begin{gathered}
+\frac{\left(w_{C} \times\left(w_{S} / w_{C}\right)+w_{S}\right) / w_{S}}{2} \\
=\frac{\left(w_{C}+w_{C}\right) / w_{C}+\left(w_{S}+w_{S}\right) / w_{S}}{2}=2,
\end{gathered}
$$

CI for $A_{C}: \frac{\lambda-2}{2-1}=\frac{2-2}{1}=0$,

where $\lambda=\frac{\left(w_{C, L}+w_{C, C} \times\left(C_{C} / C_{L}\right)\right) / w_{C, L}}{2}$

$$
\begin{gathered}
+\frac{\left(w_{C, L} \times\left(C_{L} / C_{C}\right)+w_{C, C}\right) / w_{C, C}}{2} \\
=\frac{\left(C_{C}+C_{C}\right) / C_{C}+\left(C_{L}+C_{L}\right) / C_{L}}{2}=2,
\end{gathered}
$$

CI for $A_{S}: \frac{\lambda-2}{2-1}=\frac{2-2}{1}=0$,

$$
\text { where } \begin{aligned}
\lambda= & \frac{\left(w_{S, L}+w_{S, C} \times\left(S_{L} / S_{C}\right)\right) / w_{S, L}}{2} \\
& +\frac{\left(w_{S, L} \times\left(S_{C} / S_{L}\right)+w_{S, C}\right) / w_{S, C}}{2} \\
= & \frac{\left(S_{L}+S_{L}\right) / S_{L}+\left(S_{C}+S_{C}\right) / S_{C}}{2}=2 .
\end{aligned}
$$


TABLE 8: Equipment and support.

\begin{tabular}{lll}
\hline & Demand content & Description \\
\hline \multirow{2}{*}{ Equipment } & Hardware & Notebook with Intel \\
& & Core 2 Duo CPU \\
& P7450 and 4 G RAM \\
& Microsoft Windows 7, \\
& & Microsoft Office 2007, \\
& Software & Microsoft Visual Studio \\
& & 2008, Mathematica, \\
& & and Eclipse \\
\hline
\end{tabular}

Information and

Finance of

Support Management National

Chiao Tung University

website

National Chiao Tung University website

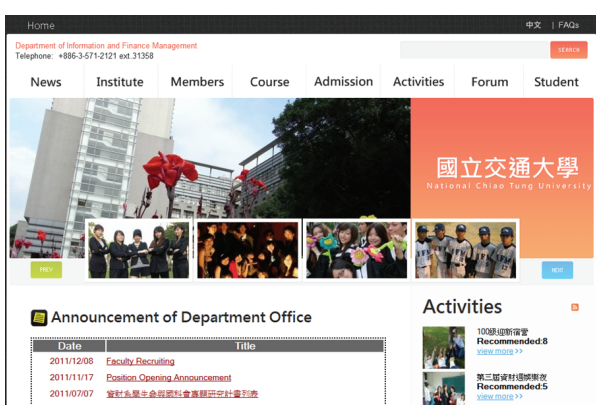

FIGURE 2: IFM web portal.

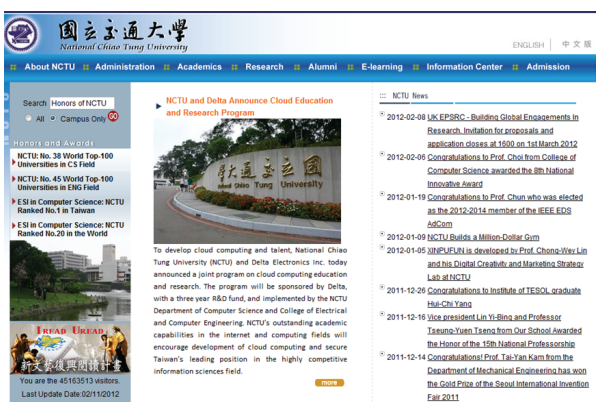

Figure 3: NCTU web portal.

4.1.1. Case 1: Information and Finance of Management at the National Chiao Tung University Website. The first case involved the Information and Finance of Management website. IFM is a department of NCTU; therefore, its website has fewer users than the NCTU website. Its web portal is shown in Figure 2. The main services provided by the IFM website include department news, institute and member introductions, course outlines, admission information, event listings, discussion forums, and information for enrolled students.

4.1.2. Case 2: The National Chiao Tung University Website. The second case involved the NCTU website; its web portal is shown in Figure 3. The university currently enrolls approximately 140,000 students. The main services provided by the NCTU website include an introduction to the institution, links to departmental overviews and websites, administrative functions, alumni services, e-learning, and campus information.

4.2. Experimental Results. The network behavior was analyzed and tested using the curve-fitting method. Based on the network behaviors, the cost and SLA were calculated using the proposed models in Section 3 to generate the criterion weights. Finally, this study used AHP to analyze the weight of each strategy for decision making.

4.2.1. Collection and Analysis of Network Behavior. This study analyzed the network behaviors to obtain the probability distribution of request arrival process and service time. The queueing model was used with these probability distributions for criterion analyses.

(1) Request Arrival Process. For the IFM case, this study used the user access records from the IFM web server for the ment and support materials used for the experiments. 


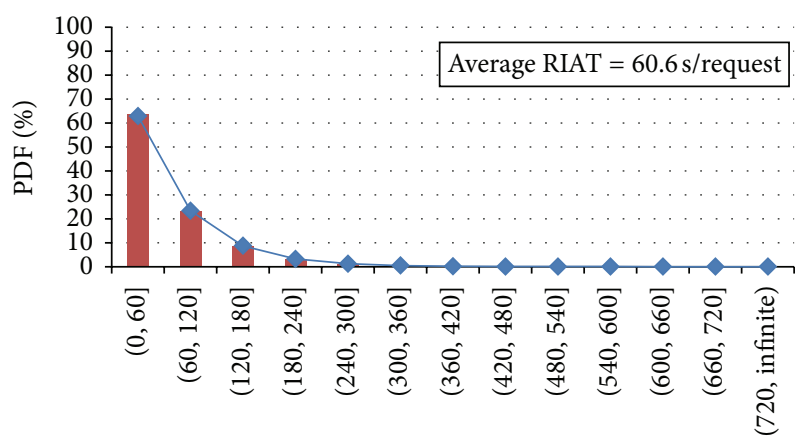

$\rightarrow \mathrm{ED}$

RIAT (s/request)

FIGURE 4: The PDF of RIAT obtained from IFM records.

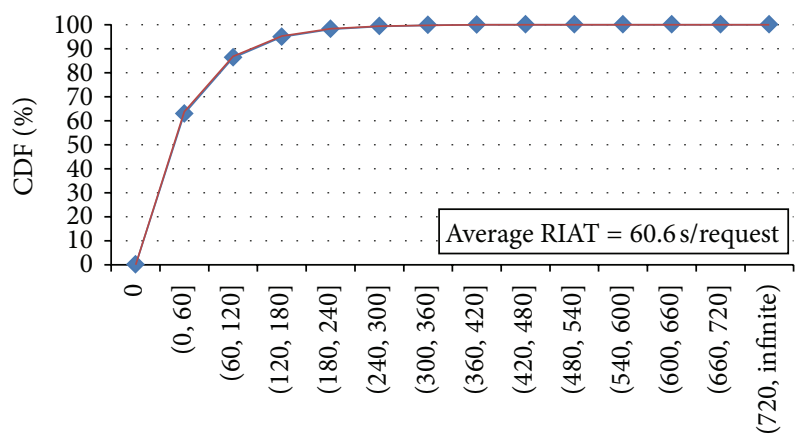

$\longrightarrow \mathrm{ED}$
$\longrightarrow \mathrm{RD}$

RIAT (s/request)

FIGURE 5: Comparison of the measured IFM request events and their corresponding cumulative probability density using the exponential distribution function (average RIAT $1 / \lambda=60.6 \mathrm{sec} /$ request).

period from March 2009 to November 2010. Subsequently, the probability density function (PDF) and the cumulative density function (CDF) of the request interarrival time (RIAT) distribution were collected and analyzed. The average RIAT $(1 / \lambda)$ was $60.6 \mathrm{sec} /$ request for the recorded request events. This study assumed that the probability distribution of the real data $(\mathrm{RD})$ is the exponential distribution (ED) function with an average RIAT of $60.6 \mathrm{sec} /$ request, as shown in Table 9 and Figures 4 and 5. The PDFs of ED and RD were defined as $E$ and $O$. The chi-square test was used to test this assumption. The test results showed that $\chi^{2}=0.0005<\chi_{12,0.05}^{2}=$ 21.026 when $\alpha=0.05$ [16], and no significant difference was observed.

For the NCTU case, the user access records during February 2011 were collected from the NCTU web server for network behavior analyses. The average RIAT was $0.1204 \mathrm{sec} /$ request for the recorded requests events, and the probability distributions were obtained, as shown in Table 10 and Figures 6 and 7 . The test results showed that $\chi^{2}=0.7105<\chi_{12,0.05}^{2}=$ 21.026 when $\alpha=0.05$ [16], and no significant difference was observed.

Therefore, this study assumed that the request arrival process corresponds to a Poisson process with requests arriving
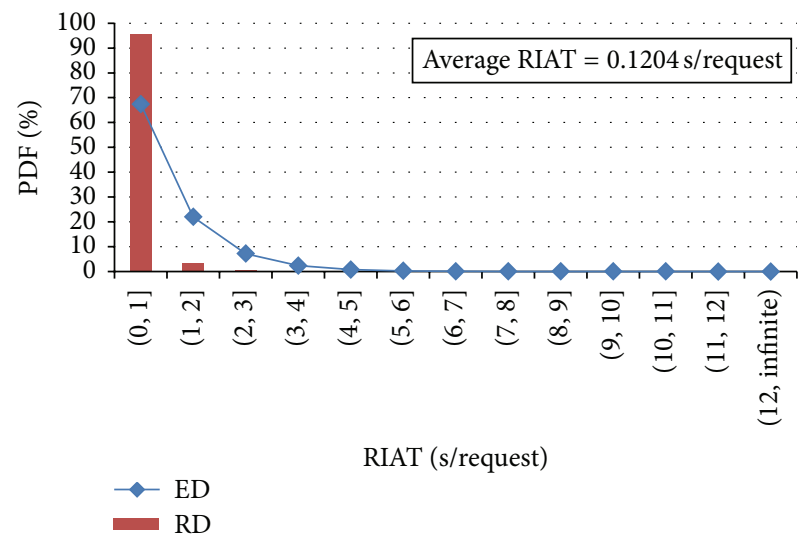

RIAT (s/request)

FIgURE 6: The PDF of RIAT obtained from IFM records.

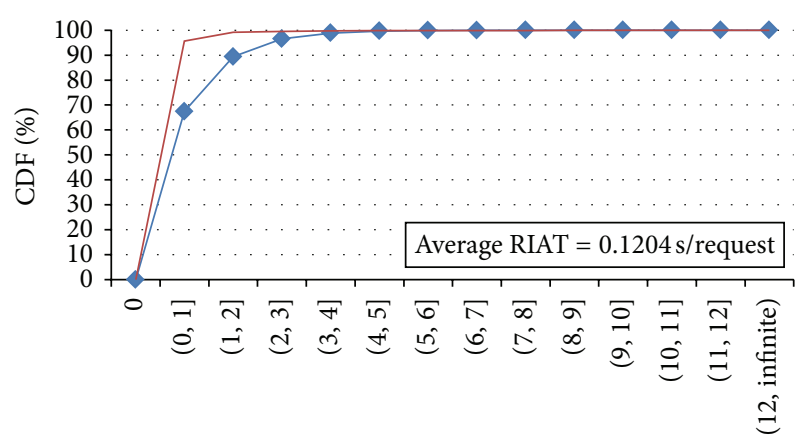

RIAT (s/request)

$\leftrightarrow$ ED

RD

FIGURE 7: Comparison of the measured NCTU request events and the corresponding cumulative probability density using the exponential distribution function (average RIAT $1 / \lambda=0.1204 \mathrm{sec} /$ request).

at rate $\lambda$ for network behavior analysis [17]. The probability density function can be defined as (14) which shows $k$ requests per day. The parameters of (14) are shown in Table 11.

Consider

$$
\operatorname{Pr}(k)=\frac{(\lambda t)^{k} e^{-\lambda t}}{k !}
$$

(2) Request Service Time. This study used the records mentioned in Section 4.2.1(1) to analyze the probability distribution of request service time. For the IFM case, the average service time $1 / \mu$ was $12.62 \mathrm{~ms} /$ request. The $\mathrm{ED}$ function fit the cumulative probability density of the service time, the average service time was $12.62 \mathrm{~ms} /$ request, as shown Table 12 and Figures 8 and 9. The test results showed that $\chi^{2}=0.0564<$ $\chi_{12,0.05}^{2}=21.026$ when $\alpha=0.05$ [16], and no significant difference was observed.

For the NCTU case, the average service time $1 / \mu$ was $24.24 \mathrm{~ms} /$ request. This study assumed that the probability of request service time is distributed as an ED. The probability distributions of request service time obtained from the NCTU records are shown in Table 13 and Figures 10 and 11. 
TABLE 9: The probability distributions of RIAT obtained from IFM records.

\begin{tabular}{|c|c|c|c|c|c|}
\hline RIAT (sec/request) & PDF of ED $(E)$ & PDF of RD $(O)$ & CDF of ED & CDF of RD & $(E-O)^{2} / E$ \\
\hline$[0,60]$ & $62.846 \%$ & $63.715 \%$ & $62.846 \%$ & $63.715 \%$ & 0.0001 \\
\hline$(60,120]$ & $23.350 \%$ & $23.119 \%$ & $86.196 \%$ & $86.834 \%$ & 0.0000 \\
\hline$(120,180]$ & $8.675 \%$ & $8.389 \%$ & $94.871 \%$ & $95.223 \%$ & 0.0001 \\
\hline$(180,240]$ & $3.223 \%$ & $3.044 \%$ & $98.094 \%$ & $98.267 \%$ & 0.0001 \\
\hline$(240,300]$ & $1.198 \%$ & $1.104 \%$ & $99.292 \%$ & $99.371 \%$ & 0.0001 \\
\hline$(300,360]$ & $0.445 \%$ & $0.401 \%$ & $99.737 \%$ & $99.772 \%$ & 0.0000 \\
\hline$(360,420]$ & $0.165 \%$ & $0.145 \%$ & $99.902 \%$ & $99.917 \%$ & 0.0000 \\
\hline$(420,480]$ & $0.061 \%$ & $0.053 \%$ & $99.964 \%$ & $99.970 \%$ & 0.0000 \\
\hline$(480,540]$ & $0.023 \%$ & $0.019 \%$ & $99.987 \%$ & $99.989 \%$ & 0.0000 \\
\hline$(540,600]$ & $0.008 \%$ & $0.007 \%$ & $99.995 \%$ & $99.996 \%$ & 0.0000 \\
\hline$(600,660]$ & $0.003 \%$ & $0.003 \%$ & $99.998 \%$ & $99.999 \%$ & 0.0000 \\
\hline$(660,720]$ & $0.001 \%$ & $0.001 \%$ & $99.999 \%$ & $100.000 \%$ & 0.0000 \\
\hline (720, infinite) & $0.001 \%$ & $0.000 \%$ & $100.000 \%$ & $100.000 \%$ & 0.0000 \\
\hline Summary & & & & & 0.0005 \\
\hline
\end{tabular}

TABLE 10: The probability distributions of RIAT obtained from NCTU records.

\begin{tabular}{|c|c|c|c|c|c|}
\hline RIAT (sec/request) & PDF of ED $(E)$ & PDF of RD $(O)$ & CDF of ED & CDF of RD & $(E-O)^{2} / E$ \\
\hline$[0,1]$ & $67.386 \%$ & $95.665 \%$ & $67.386 \%$ & $95.665 \%$ & 0.1187 \\
\hline$(1,2]$ & $21.977 \%$ & $3.474 \%$ & $89.363 \%$ & $99.139 \%$ & 0.1558 \\
\hline$(2,3]$ & $7.168 \%$ & $0.412 \%$ & $96.531 \%$ & $99.550 \%$ & 0.0637 \\
\hline$(3,4]$ & $2.338 \%$ & $0.173 \%$ & $98.869 \%$ & $99.724 \%$ & 0.0200 \\
\hline$(4,5]$ & $0.762 \%$ & $0.074 \%$ & $99.631 \%$ & $99.797 \%$ & 0.0062 \\
\hline$(5,6]$ & $0.249 \%$ & $0.045 \%$ & $99.880 \%$ & $99.842 \%$ & 0.0017 \\
\hline$(6,7]$ & $0.081 \%$ & $0.035 \%$ & $99.961 \%$ & $99.877 \%$ & 0.0003 \\
\hline$(7,8]$ & $0.026 \%$ & $0.019 \%$ & $99.987 \%$ & $99.896 \%$ & 0.0000 \\
\hline$(8,9]$ & $0.009 \%$ & $0.015 \%$ & $99.996 \%$ & $99.911 \%$ & 0.0000 \\
\hline$(9,10]$ & $0.003 \%$ & $0.012 \%$ & $99.999 \%$ & $99.923 \%$ & 0.0003 \\
\hline$(10,11]$ & $0.001 \%$ & $0.009 \%$ & $100.000 \%$ & $99.933 \%$ & 0.0008 \\
\hline$(11,12]$ & $0.000 \%$ & $0.010 \%$ & $100.000 \%$ & $99.942 \%$ & 0.0030 \\
\hline (12, infinite) & $0.000 \%$ & $0.058 \%$ & $100.000 \%$ & $100.000 \%$ & 0.3400 \\
\hline Summary & & & & & 0.7105 \\
\hline
\end{tabular}

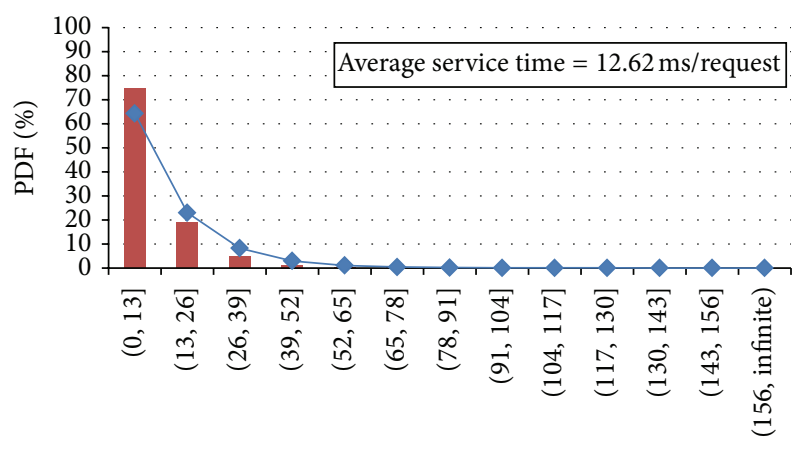

Service time (ms/request)

$$
\neg \mathrm{ED}
$$

FIGURE 8: The PDF of request service time obtained from IFM records.

The test results showed that $\chi^{2}=0.6354<\chi_{12,0.05}^{2}=$ 21.026 when $\alpha=0.05$ [16], and no significant difference was observed.
TABLE 11: Parameters of the request arrival process.

\begin{tabular}{|c|c|}
\hline Parameter & Description \\
\hline $\operatorname{Pr}(k)$ & $\begin{array}{l}\text { Probability for a number of requests arriving } \\
\text { in a cycle } t\end{array}$ \\
\hline$\lambda$ (request $/ \mathrm{min})$ & Average request arrival rate \\
\hline$t(\min )$ & Request cycle time \\
\hline$k$ (requests) & $\begin{array}{c}\text { Number of arriving requests per current } \\
\text { cycle time }\end{array}$ \\
\hline
\end{tabular}

(3) Summary. This study showed that the request arrival process is a Poisson process and the service time is distributed as ED. Therefore, the service process can be modeled using a simple $\mathrm{M} / \mathrm{M} / \mathrm{c} / \mathrm{c}$ queue with the parameters $\lambda$ and $1 / \mu[18]$. Figure 12 shows a state transition rate diagram of the service process, where state $c$ denotes that there are $c$ clients in the server, and $P_{i}$ denotes the probability of state $i$. The $\operatorname{SBR} \operatorname{Pr}_{l}(c)$ in (5) was derived in (15), where $c$ denotes the maximum number of connections that are simultaneously processed by a traditional local server. 
TABLE 12: The probability distributions of request service time obtained from IFM records.

\begin{tabular}{|c|c|c|c|c|c|}
\hline Service time (ms/request) & PDF of ED $(E)$ & PDF of RD $(O)$ & CDF of ED & CDF of RD & $(E-O)^{2} / E$ \\
\hline 0 & $0.00 \%$ & $0.00 \%$ & $0.00 \%$ & $0.00 \%$ & \\
\hline$[0,13]$ & $64.30 \%$ & $74.60 \%$ & $64.30 \%$ & $74.60 \%$ & 0.0165 \\
\hline$(13,26]$ & $22.95 \%$ & $18.95 \%$ & $87.26 \%$ & $93.55 \%$ & 0.0070 \\
\hline$(26,39]$ & $8.19 \%$ & $4.81 \%$ & $95.45 \%$ & $98.36 \%$ & 0.0140 \\
\hline$(39,52]$ & $2.92 \%$ & $1.22 \%$ & $98.38 \%$ & $99.58 \%$ & 0.0099 \\
\hline$(52,65]$ & $1.04 \%$ & $0.31 \%$ & $99.42 \%$ & $99.89 \%$ & 0.0052 \\
\hline$(65,78]$ & $0.37 \%$ & $0.08 \%$ & $99.79 \%$ & $99.97 \%$ & 0.0023 \\
\hline$(78,91]$ & $0.13 \%$ & $0.02 \%$ & $99.93 \%$ & $99.99 \%$ & 0.0010 \\
\hline$(91,104]$ & $0.05 \%$ & $0.01 \%$ & $99.97 \%$ & $100.00 \%$ & 0.0003 \\
\hline$(104,117]$ & $0.02 \%$ & $0.00 \%$ & $99.99 \%$ & $100.00 \%$ & 0.0002 \\
\hline$(117,130]$ & $0.01 \%$ & $0.00 \%$ & $100.00 \%$ & $100.00 \%$ & 0.0001 \\
\hline$(130,143]$ & $0.00 \%$ & $0.00 \%$ & $100.00 \%$ & $100.00 \%$ & 0.0000 \\
\hline$(143,156]$ & $0.00 \%$ & $0.00 \%$ & $100.00 \%$ & $100.00 \%$ & 0.0000 \\
\hline$(156$, infinite $)$ & $0.00 \%$ & $0.00 \%$ & $100.00 \%$ & $100.00 \%$ & 0.0000 \\
\hline Summary & & & & & 0.0564 \\
\hline
\end{tabular}

TABLE 13: The probability distributions of request service time obtained from NCTU records.

\begin{tabular}{|c|c|c|c|c|c|}
\hline Service time (ms/request) & PDF of ED $(E)$ & PDF of RD $(O)$ & CDF of ED & $\mathrm{CDF}$ of $\mathrm{RD}$ & $(E-O)^{2} / E$ \\
\hline$[0,12]$ & $39.046 \%$ & $76.060 \%$ & $39.046 \%$ & $76.060 \%$ & 0.3509 \\
\hline$(12,24]$ & $23.800 \%$ & $18.210 \%$ & $62.846 \%$ & $94.270 \%$ & 0.0131 \\
\hline$(24,36]$ & $14.507 \%$ & $4.360 \%$ & $77.353 \%$ & $98.630 \%$ & 0.0710 \\
\hline$(36,48]$ & $8.843 \%$ & $1.040 \%$ & $86.196 \%$ & $99.670 \%$ & 0.0688 \\
\hline$(48,60]$ & $5.390 \%$ & $0.250 \%$ & $91.586 \%$ & $99.920 \%$ & 0.0490 \\
\hline$(60,72]$ & $3.285 \%$ & $0.060 \%$ & $94.871 \%$ & $99.980 \%$ & 0.0317 \\
\hline$(72,84]$ & $2.003 \%$ & $0.020 \%$ & $96.874 \%$ & $100.000 \%$ & 0.0196 \\
\hline$(84,96]$ & $1.221 \%$ & $0.000 \%$ & $98.094 \%$ & $100.000 \%$ & 0.0122 \\
\hline$(96,108]$ & $0.744 \%$ & $0.000 \%$ & $98.838 \%$ & $100.000 \%$ & 0.0074 \\
\hline$(108,120]$ & $0.454 \%$ & $0.000 \%$ & $99.292 \%$ & $100.000 \%$ & 0.0045 \\
\hline$(120,132]$ & $0.276 \%$ & $0.000 \%$ & $99.568 \%$ & $100.000 \%$ & 0.0028 \\
\hline$(132,144]$ & $0.169 \%$ & $0.000 \%$ & $99.737 \%$ & $100.000 \%$ & 0.0017 \\
\hline (144, infinite) & $0.263 \%$ & $0.000 \%$ & $100.000 \%$ & $100.000 \%$ & 0.0026 \\
\hline Summary & & & & & 0.6354 \\
\hline
\end{tabular}

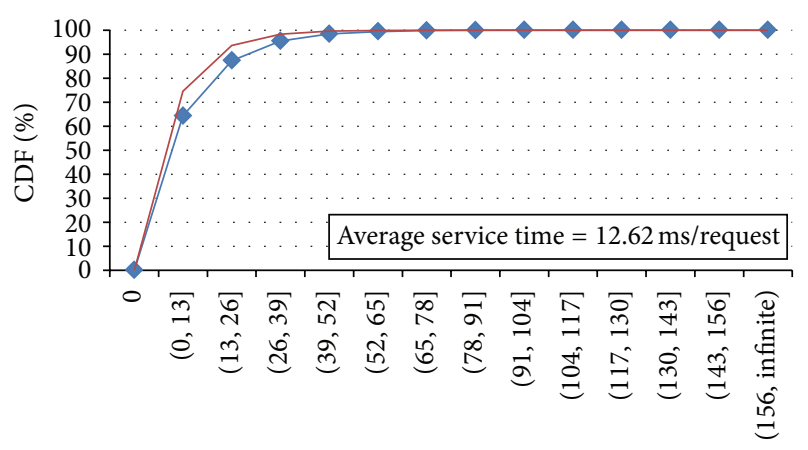

Service time (ms/request)

$$
\begin{aligned}
& \multimap \mathrm{ED} \\
& -\mathrm{RD}
\end{aligned}
$$

Figure 9: Comparison of the measured IFM service time and its corresponding cumulative probability density using the exponential distribution function (average service time $1 / \mu=12.62 \mathrm{~ms}$ ).

\section{Consider}

$$
\text { Let } \begin{aligned}
\sum_{i=0}^{c} P_{i}=1 & \Longrightarrow P_{k}=\frac{1}{k !}\left(\frac{\lambda}{\mu}\right)^{k} P_{0} \quad \forall \rho=\frac{\lambda}{\mu} \\
& \Longrightarrow P_{c}=\frac{\rho^{c}}{c !} P_{0}=\frac{\rho^{c} / c !}{\sum_{k=0}^{c}\left(\rho^{k} / k !\right)}=\operatorname{Pr}_{l}(c)=B_{L} .
\end{aligned}
$$

4.2.2. Analysis of Criteria. The cost and SLA were regarded as the criteria, and the analyzed results are presented in this section.

(1) Criterion 1: Cost. The proposed equations were used to calculate the costs of a traditional local server versus a PaaS platform. The costs were calculated using GAE, for which 


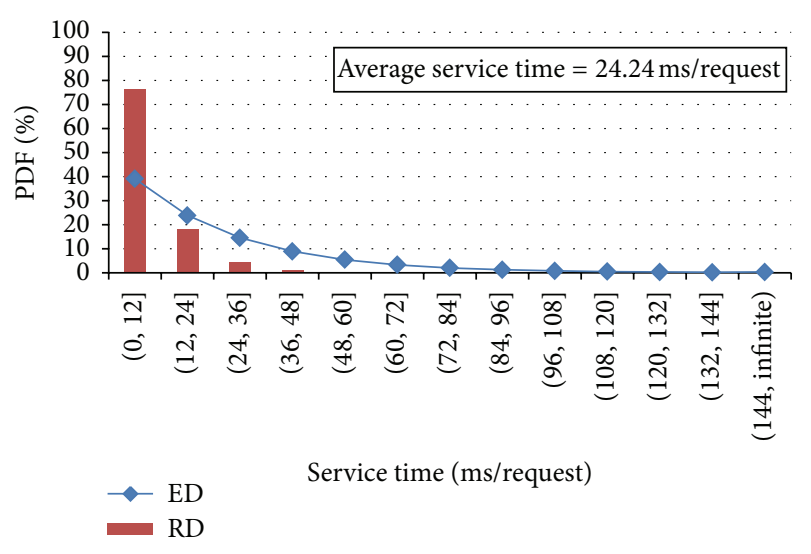

FIGURE 10: The PDF of request service time obtained from NCTU records.

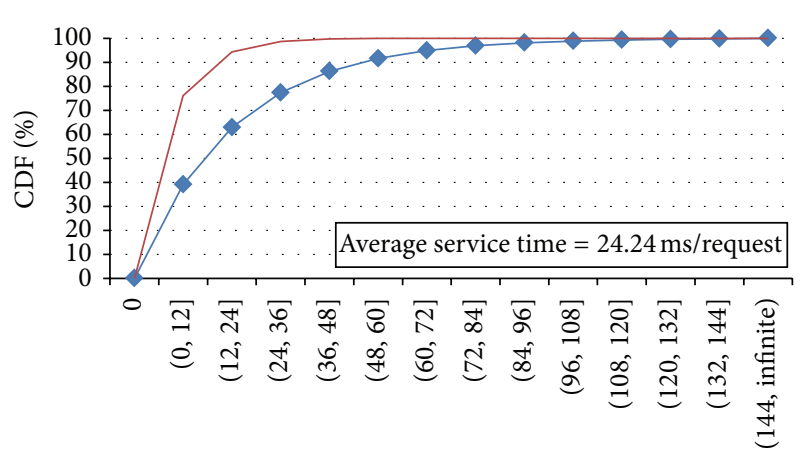

$$
\longrightarrow \text { ED }
$$

Service time (ms/request)

FIGURE 11: Comparison of the measured NCTU service time and its corresponding cumulative probability density using the exponential distribution function (average service time $1 / \mu=24.24 \mathrm{~ms}$ ).

people are charged according to the billing quota, as shown in Tables 2 and 3 in Section 2.

(a) Local Server Costs. Based on (3), the costs include network costs, electricity, server depreciation expenses, and personnel costs. The price of a server was assumed to be NT 60,000 depreciated over 5 years; therefore, the depreciation was $C_{\mathrm{SDE}}=\mathrm{NT} 1,000$ per month. Electricity costs are based on the Taiwan Power Company and electricity kilowatt-hour (KWH) formula, shown in (16):

$$
\mathrm{KWH}=\frac{\text { electric power }(\text { watts }) \times \text { hours }}{1,000} .
$$

Regarding power consumption, Barroso [19] indicated a typical power use of 200 Watts for low-end servers; therefore, the $\mathrm{KWH}$ value was calculated as 200 (watts) $\times 24 \times 30 / 1,000=$ 144. The electricity cost was based on the Electricity Tariffs Table [20] and calculated on a per-month basis using (17):

$$
C_{E}=144 \times 2.68=385.92(\mathrm{NT} / \mathrm{month}) .
$$

The network costs were omitted because both websites are in the university network. A student was hired to maintain
TABLE 14: Cost parameter data for CCS.

\begin{tabular}{lc}
\hline Parameter & Real data \\
\hline$\xi$ & $11793 \times 2^{-30} \mathrm{~GB}$ \\
$r$ & $43,200,000$ (requests) \\
$k$ & $0 \sim 43,200,000$ (requests) \\
$\lambda$ & 0.7635 (requests/sec) in IFM \\
$t$ & 8.3 (requests/sec) in NCTU \\
$C_{G}$ & $86400(\mathrm{sec})$ \\
\hline
\end{tabular}

this web server at a salary of 3000 (NT/month). The total cost of a traditional local server is shown in (18):

$$
\begin{aligned}
C_{L} & =C_{N}+C_{E}+C_{\mathrm{SDE}}+C_{P} \\
& =0+386+1,000+3,000=4,386(\mathrm{NT}) .
\end{aligned}
$$

(b) PaaS Platform Costs. The probability density functions for the NCTU and IFM websites are shown in (14). Thus, (19) is derived to calculate the costs of both $\left(C_{C}\right)$ in accordance with (4) if they operate on a PaaS platform.

Consider

$$
C_{C}=\left(\left\lceil\xi \times \sum_{k=0}^{r} k \times \frac{(\lambda t)^{k} e^{-\lambda t}}{k !}\right\rceil-1\right) \times C_{G} .
$$

This study used actual data from the NCTU and IFM websites to calculate the cost for the GAE PaaS platform. The incoming bandwidth cost effects were ignored because the incoming bandwidth requirements were low. The actual data shown in Table 14 were substituted for the parameters.

The bandwidth cost per day of the NCTU website on the PaaS platform was calculated using (20):

$$
\begin{aligned}
C_{C}= & \left(\left\lceil\xi \times \sum_{k=0}^{r} k \times \frac{(\lambda t)^{k} e^{-\lambda t}}{k !}\right]-1\right) \times C_{G} \\
= & \left(\left[11,793 \times 2^{-30} \times \sum_{k=0}^{43,200,000} k\right.\right. \\
& \left.\left.\times \frac{(8.3 \times 86,400)^{k} \times e^{-8.3 \times 86,400}}{k !}\right]-1\right)
\end{aligned}
$$$$
\times \$ 0.12=\$ 0.84 \text {. }
$$

Based on the spot exchange rate of the US dollar to approximately 29 Taiwan dollars, the monthly cost of the NCTU website is $0.84 \times 29 \times 30=730.8(\mathrm{NT})$. A comparison of $C_{C}$ and $C_{L}$ indicates that if the NCTU website is deployed in the cloud, the NCTU can save $83.34 \%$ of its current costs.

(c) Numerical Analysis. Sensitivity analyses of two parameters, $C_{C}$ and $C_{L}$, and their rates of change with the change parameters were investigated as follows.

Effect of $\xi$ on $C_{C}$ and $C_{L}$. Figure 13 shows the cost $C_{C}$ against $\xi$, which indicates that $C_{C}$ increases as the amount of data per 


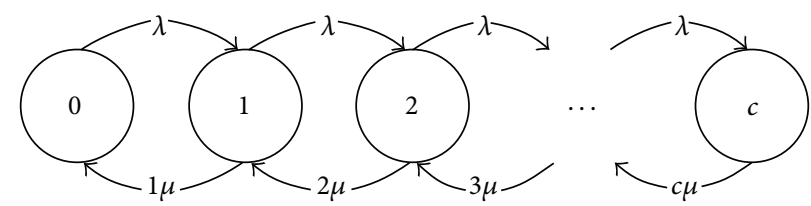

FIGURE 12: State transition rate diagram for the service scheme.

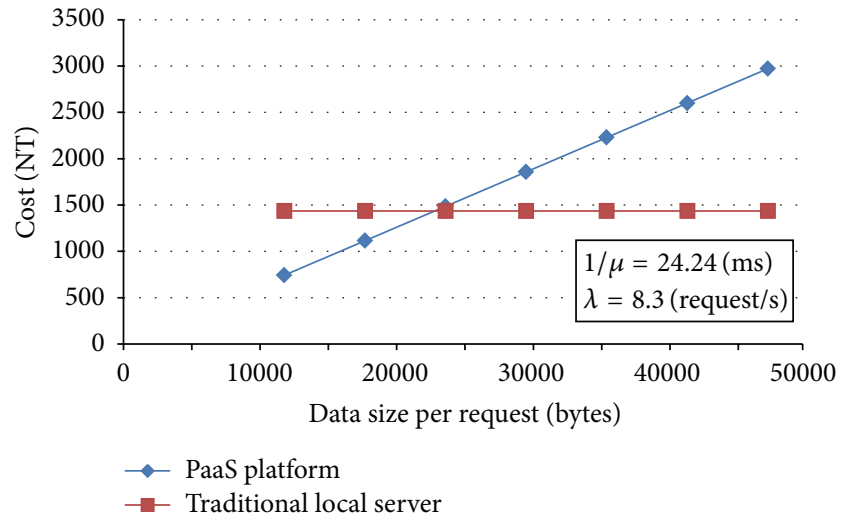

Figure 13: Effect of $\xi$ on $C_{C}$ and $C_{L}$.

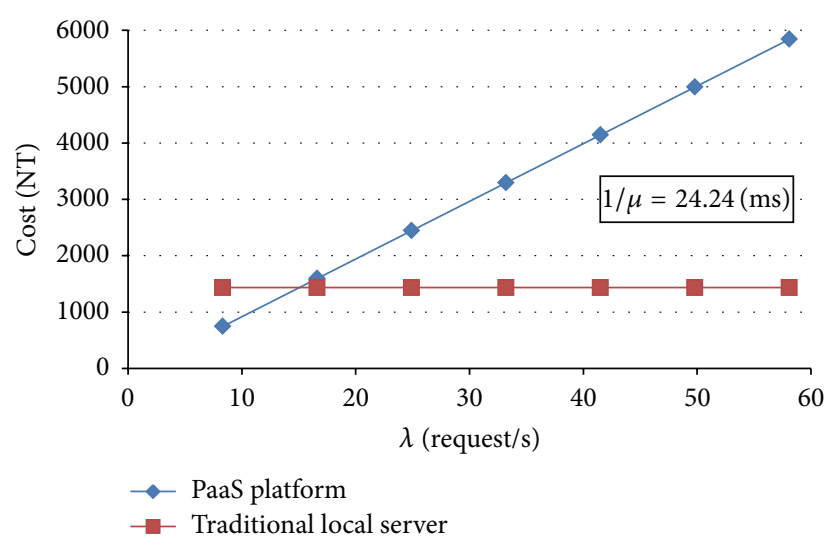

Figure 14: Effect of $\lambda$ on $C_{C}$ and $C_{L}$.

request $\xi$ increases. This phenomenon is explained as follows: when the amount of data per request increases, the cost $C_{C}$ is higher in the PaaS platform. Regardless of the amount of data per request changes, the cost $C_{L}$ for a traditional local server is not influenced. Figure 13 shows the importance of accounting for the amount of data per request when seeking cost reductions on a PaaS platform.

Effect of $\lambda$ on $C_{C}$ and $C_{L}$. Figure 14 shows the cost $C_{C}$ against $\lambda$, which indicates that $C_{C}$ increases as the average request arrival rate $\lambda$ increases. This phenomenon is explained as follows: as the average request arrival rate $\lambda$ increases, the cost $C_{C}$ in the PaaS platform increases. Conversely, regardless of whether the average request arrival rate $\lambda$ changes, the $\operatorname{cost} C_{L}$ is not influenced in a traditional local server setup. Figure 14 shows the importance of the average request arrival rate $\lambda$ in reducing costs on the PaaS platform.
TABLE 15: SLA parameter data for a traditional local server.

\begin{tabular}{lc}
\hline Parameter & Real data \\
\hline$\lambda$ & 0.0165 (requests/sec) for IFM \\
& 8.3 (requests/sec) for NCTU \\
$1 / \mu$ & $12.62(\mathrm{~ms})$ for IFM \\
& $24.24(\mathrm{~ms})$ for NCTU \\
$\rho$ & 0.00021 for IFM \\
$c$ & 0.201 for NCTU \\
\end{tabular}

(2) Criterion 2: Service Level Agreement. This study also examined the SLA in accordance with SBR. When a service provider cannot manage a large number of simultaneous users, service errors or an inability to complete the services occur, which may result in a loss of customers. Therefore, the SBR for traditional local servers and the PaaS platform was calculated based on the previously derived equations.

(a) SLA for Local Servers. The majority of website developers use the Apache HTTP Server to build their websites. In the Apache HTTP Server, the directive sets the limit on the number of simultaneous requests that can be served, with a default value of 256. Therefore, it is assumed that $c$ is equal to 256 , which is the maximum number of connections that can be processed simultaneously.

In accordance with the analytic models, the service process can be modeled using a simple $\mathrm{M} / \mathrm{M} / \mathrm{c} / \mathrm{c}$ queue with the parameters $\lambda$ and $1 / \mu$, as shown in (15). The SLA can be calculated using (6) and (15). The parameters obtained from real data for the IFM and NCTU websites are shown in Table 15. Therefore, (21) and (22) are used to calculate the SLAs of the IFM and NCTU websites, respectively, for a local server.

Consider

$$
\begin{aligned}
B_{L} & =\operatorname{Pr}_{l}(c)=\frac{0.00021^{256} / 256 !}{\sum_{k=0}^{256}\left(0.00021^{k} / k !\right)}=3.586 \times 10^{-1449} \\
& \Longrightarrow S_{L}=1-B_{L}=1-3.586 \times 10^{-1449} \\
B_{L} & =\operatorname{Pr}_{l}(c)=\frac{0.201^{256} / 256 !}{\sum_{k=0}^{256}\left(0.201^{k} / k !\right)}=3.958 \times 10^{-686} \\
& \Longrightarrow S_{L}=1-B_{L}=1-3.958 \times 10^{-686}
\end{aligned}
$$

(b) SLA for PaaS Platforms. For CCS, the probability that the maxima possible requests are exceeded in 1 minute was calculated; the SLA for the PaaS platform can be obtained by using (6) and (14). The actual data parameters are shown in Table 16. Equations (23) and (24) were subsequently used to 
TABLE 16: SLA parameter data for CCS.

\begin{tabular}{lc}
\hline Parameter & Real data \\
\hline$\lambda$ & 0.0165 (requests/sec) for IFM \\
$t$ & 8.3 (requests/sec) for NCTU \\
$n$ & $60(\mathrm{sec})$ \\
\hline
\end{tabular}

calculate the SLAs of the IFM and NCTU websites in the PaaS platform, respectively.

Consider

$$
\begin{aligned}
B_{C} & =\sum_{k=n+1}^{\infty} \frac{(\lambda t)^{k} e^{-\lambda t}}{k !} \\
& =\sum_{k=45200+1}^{\infty} \frac{(0.0165 \times 60)^{k} e^{-0.0165 \times 60}}{k !} \\
& =1.110 \times 10^{-16} \\
& \Longrightarrow S_{C}=1-B_{C}=1-1.110 \times 10^{-16}, \\
B_{C} & =\sum_{k=n+1}^{\infty} \frac{(\lambda t)^{k} e^{-\lambda t}}{k !} \\
& =\sum_{k=45200+1}^{\infty} \frac{(8.3 \times 60)^{k} e^{-8.3 \times 60}}{k !}=10^{-10} \\
& \Longrightarrow S_{C}=1-B_{C}=1-10^{-10} .
\end{aligned}
$$

(c) Numerical Analysis. A numerical analysis of the models was used to investigate the network behavior of the RAIT, amount of data, and service time. It was assumed that the arrival rate has an ED with a mean of $\lambda$ and the service time has an ED with a mean of $1 / \mu$. The change in $B_{C}$ and $B_{L}$ was examined using the change parameters. The effects of the input parameters were examined as follows.

Effect of $\lambda$ on $S_{C}$ and $S_{L}$. Figure 15 shows the SLA $S_{C}$ and $S_{L}$ against $\lambda$, and indicates that $S_{C}$ and $S_{L}$ increase as the average request arrival rate $\lambda$ increases. This phenomenon is explained as follows: as the average request arrival rate $\lambda$ increases, the SLA $S_{C}$ and $S_{L}$ for both the PaaS platform and the local server increases. The effect of $\lambda$ on $S_{C}$ is more important than the effect of $\lambda$ on $S_{L}$. For an average arrival rate of $\lambda=750 \mathrm{request} / \mathrm{sec}$, the SLA decreases rapidly in the PaaS platform. By contrast, on a traditional local server, the SLA decreases slowly until an average arrival rate of $\lambda=8000 \mathrm{request} / \mathrm{sec}$ is achieved. Although cloud computing has an infinite capacity, the PaaS platform is limited by its maximum rate quota. Figure 15, shows the importance of the average arrival rate $\lambda$ in understanding the SLA on the PaaS platform versus a local server.

Effect of $1 / \mu$ on $S_{C}$ and $S_{L}$. Figure 16 shows the SLA $S_{C}$ against $1 / \mu$, which indicates that $S_{L}$ decreases as the average service time $1 / \mu$ increases. This phenomenon is explained as follows:

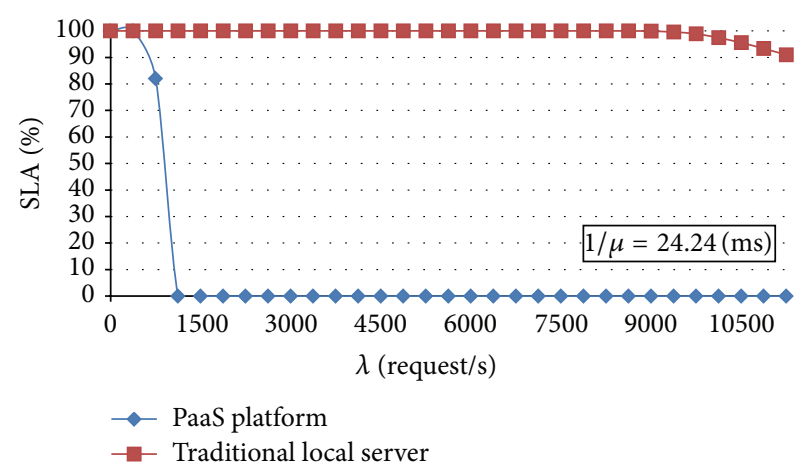

Figure 15: Effect of $\lambda$ on $S_{C}$ and $S_{L}$.

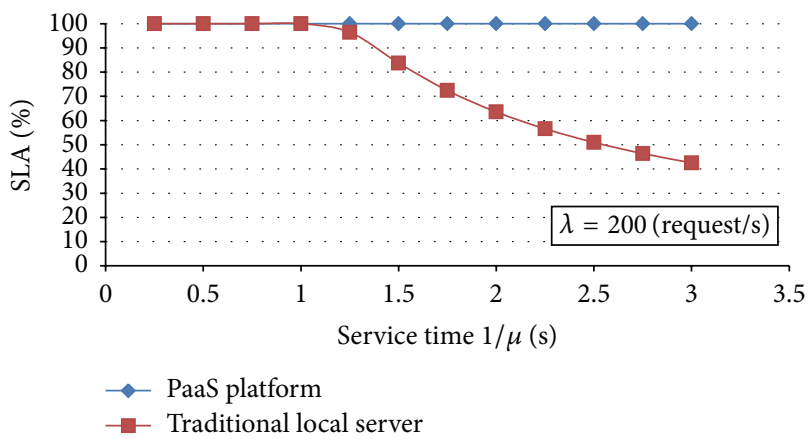

Figure 16: Effect of $1 / \mu$ on $S_{C}$ and $S_{L}$.

as the average service time $1 / \mu$ increases, the $\operatorname{SBR} S_{L}$ decreases in the traditional local server. Conversely, regardless of the manner in which the average service time $1 / \mu$ changes, the SLA $S_{C}$ has no effect in the PaaS platform. Figure 16 shows that the average service time $1 / \mu$ is a crucial factor in reducing the SLA on a local server. When the average service time is long, moving to a service that deploys on a PaaS platform may mitigate or avoid the SBR problem.

4.2.3. Analysis of Deployment Strategy. For deployment strategy decision making, AHP is used to calculate the score of each strategy in this section. In accordance with (19), (20), (22), and (24), this study used (13) and a number of parameters to calculate the value of $\delta$ as (25) for the NCTU website deployment. The parameters were as follows: $C_{L}=$ 4386, $C_{C}=730.8, S_{L}=1-3.958 \times 10^{-686}$, and $S_{C}=$ $1-10^{-10}$. The results show that the value of $\delta$ is equal to $6.99934 \times 10^{-11}$ and $S_{C}$ is larger than 0.99 . Therefore, the NCTU website would be suitable for deployment as a CCS in the PaaS platform if the website administrator assumes that the weight of cost $w_{C}$ is equal to the weight of SLA $w_{S}$ (26). Because $S_{L}$ is similar to $S_{C}$, the SLA has a small effect on strategy decision making. It is possible to deploy in a traditional local server when $w_{S}$ must be larger than $\left(w_{C} \times 10^{11}\right) / 6.99934($ see $(27))$. For the IFM case, the value of $\delta$ was smaller than that in the NCTU case because the cost of IFM website was lower than that of the NCTU website and the SLA of the IFM website is higher than that of the 
NCTU website. Therefore, the IFM website was also suitable for deployment as a CCS in the PaaS platform.

Consider

$$
\begin{aligned}
\delta= & \frac{\left(C_{L}+C_{C}\right)\left(S_{L}-S_{C}\right)}{\left(C_{L}-C_{C}\right)\left(S_{L}+S_{C}\right)} \\
& =\frac{(4386+730.8)\left[\left(1-3.958 \times 10^{-686}\right)-\left(1-10^{-10}\right)\right]}{(4386-730.8)\left[\left(1-3.958 \times 10^{-686}\right)+\left(1-10^{-10}\right)\right]} \\
& =6.99934 \times 10^{-11},
\end{aligned}
$$

$$
\begin{gathered}
\frac{w_{C}}{w_{S}}=1>\delta=6.99934 \times 10^{-11}, \\
\frac{w_{C}}{w_{S}}<\delta=6.99934 \times 10^{-11} \Longrightarrow w_{S}>\frac{w_{C} \times 10^{11}}{6.99934} .
\end{gathered}
$$

4.3. Discussion. This study used two websites to simulate and analyze the cost and SLA of deploying services using GAE. The mathematical models based on AHP presented in Section 3 were used to analyze the cost and SLA for the PaaS platform and a traditional local server. The results show that if the NCTU website is deployed using GAE, the university would save over $83.34 \%$ of its current expenditures compared to deploying on a traditional local server; this also has a negligible low effect on the SLA. This shows that the NCTU web service is appropriate for cloud computing, and its subsidiary website is suitable for IFM.

A simulation analysis from a cost perspective shows that when the amount of data per request and average request arrival rate $\lambda$ increase, the cost $C_{C}$ in the PaaS platform is higher. Conversely, variations in the amount of data per request or $\lambda$ have no influence on the cost $C_{L}$ in a traditional local server. The results also show that as user demand on the network increases, the importance of SLA decreases, and deployment in the cloud may help to reduce average service times.

\section{Conclusions and Future Work}

This paper proposes an analytical framework that uses the curve-fitting method and analyzes user access records from the web server to generate the network behavior patterns for analyses of cost and SLA. Furthermore, this framework uses AHP to calculate the score of deploying on the PaaS platform and deploying on a traditional local server for decision making. When user demands are low, deploying services in the cloud costs less than deploying them on a traditional local server. Because it is more difficult to collect the records from business websites, academic websites which including the IFM and NCTU websites were selected as test cases and used to simulate and analyze the costs and SLA of deploying services in GAE. The results show that if the NCTU website is deployed using GAE, the university would save over $83.34 \%$ of its current expenditures than by deploying in a traditional local server; this also has a negligible effect on the SLA. However, the simulation analysis showed that, as user demand on the network increases, the importance of SLA decreases, and deploying in the cloud can help to reduce the average service time.

In future studies, the authors plan to broaden their research criteria and analyze the uses of CPU time and time spent on accessing databases; for example, various types of services have various probability density functions. These services must be further investigated in subsequent studies. In addition, the business website administrators can use the proposed framework to analyze their website records and select an optimal strategy for CCS deployments.

\section{Acknowledgment}

This study was supported by the National Science Council of Taiwan under Grants nos. NSC 100-2410-H009-039-SS2, NSC 101-2420-H-009-004-DR, and NSC 102-2410-H-009052-MY3.

\section{References}

[1] L. M. Vaquero, L. Rodero-Merino, J. Cceres, and M. Lindner, "A break in the clouds: towards a cloud definition," ACM SIGCOMM Computer Communication Review, vol. 39, no. 1, pp. 50$55,2009$.

[2] C. H. Chen, B. Y. Lin, H. C. Chang, and C. C. Lo, "The novel positioning algorithm based on cloud computing - a case study of intelligent transportation systems," Information, vol. 15, pp. 4519-4524, 2012.

[3] P. Mell and T. Grance, The NIST Definition of Cloud Computing, National Institute of Standards and Technology Special Publication 800-145, 2011.

[4] S. Subashini and V. Kavitha, "A survey on security issues in service delivery models of cloud computing," Journal of Network and Computer Applications, vol. 34, no. 1, pp. 1-11, 2011.

[5] G. Lawton, "Developing software online with platform-as-aservice technology," Computer, vol. 41, no. 6, pp. 13-15, 2008.

[6] Google App Engine, "What is google app engine," Google Inc., 2012, http://code.google.com/intl/en/appengine/docs/whatisgoogleappengine.html.

[7] M. Armbrust, A. Fox, R. Griffith et al., "A view of cloud computing," Communications of the ACM, vol. 53, no. 4, pp. 50-58, 2010.

[8] A. Bisong and S. M. Rahman, "An overview of the security concerns in enterprise network security," International Journal of Network Security and Its Applications, vol. 3, pp. 30-45, 2011.

[9] J. C. Mogul, "Network Behavior of a Busy Web Server and its Clients," Tech. Rep. 95/5, Digital Equipment Corporation, Western Research Laboratory, 1995.

[10] J. Kang and K. M. Sim, "Cloudle: a multi-criteria cloud service search engine," in Proceedings of the IEEE Asia-Pacific Services Computing Conference (APSCC '10), pp. 339-346, Hangzhou, China, December 2010.

[11] E. Deelman, G. Singh, M. Livny, B. Berriman, and J. Good, "The cost of doing science on the cloud: the montage example," in Proceedings of the International Conference for High Performance Computing, Networking, Storage and Analysis (SC '08), pp. 1-12, Austin, Tex, USA, November 2008.

[12] T. L. Saaty, "A scaling method for priorities in hierarchical structures," Journal of Mathematical Psychology, vol. 15, no. 3, pp. 234-281, 1977. 
[13] G. H. Tzeng and J. J. Huang, Multiple Attribute Decision Making: Methods and Applications, CRC Press, Taylor \& Francis Group, New York, NY, USA, 2011.

[14] T. L. Saaty, "How to make a decision: the analytic hierarchy process," European Journal of Operational Research, vol. 48, no. 1, pp. 9-26, 1990.

[15] C. H. Chen, H. C. Chang, C. Y. Su, C. C. Lo, and H. F. Lin, "Traffic speed estimation based on normal location updates and call arrivals from cellular networks," Simulation Modelling Practice and Theory, vol. 35, pp. 26-33, 2013.

[16] H. C. Chang, C. H. Chen, B. Y. Lin, H. Y. Kung, and C. C. Lo, "Traffic information estimation using periodic location update events," International Journal of Innovative Computing, Information and Control, vol. 9, pp. 2031-2041, 2013.

[17] B. Y. Lin, C. H. Chen, H. C. Chang, and C. C. Lo, "A network behavior analysis system for cloud computing service," Information, vol. 14, pp. 931-938, 2011.

[18] Y. B. Lin, M. H. Tsai, H. W. Dai, and Y. K. Chen, "Bearer reservation with preemption for voice call continuity," IEEE Transactions on Wireless Communications, vol. 8, no. 5, pp. 2716-2725, 2009.

[19] L. A. Barroso, "The price of performance: an economic case for chip multiprocessing," ACM Queue, vol. 3, pp. 49-53, 2005.

[20] Taiwan Power Company, "The electricity tariffs table," 2012, http://www.taipower.com.tw/content/q_service/images/main_3 _6_3.pdf. 


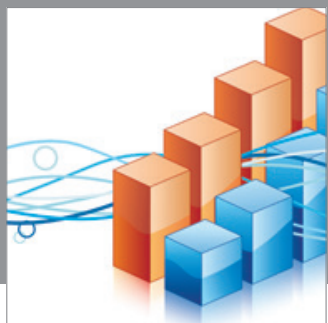

Advances in

Operations Research

mansans

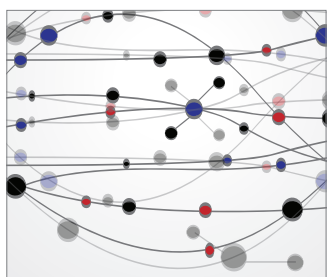

The Scientific World Journal
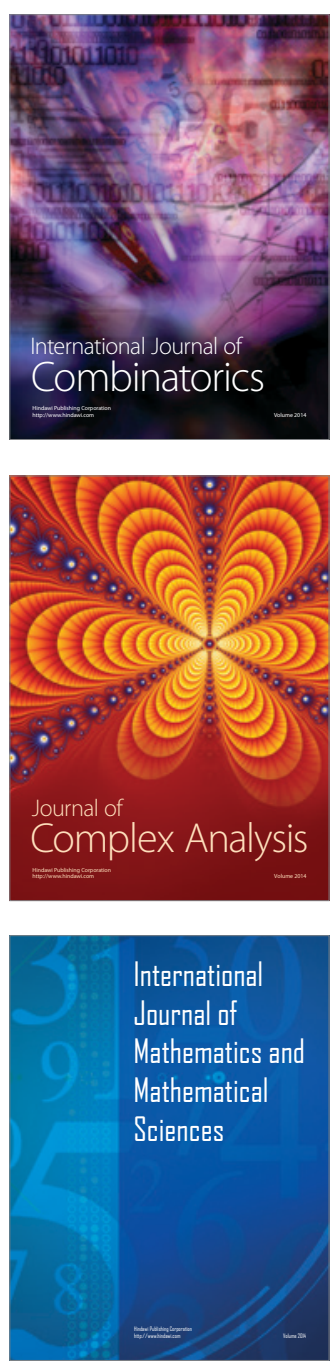
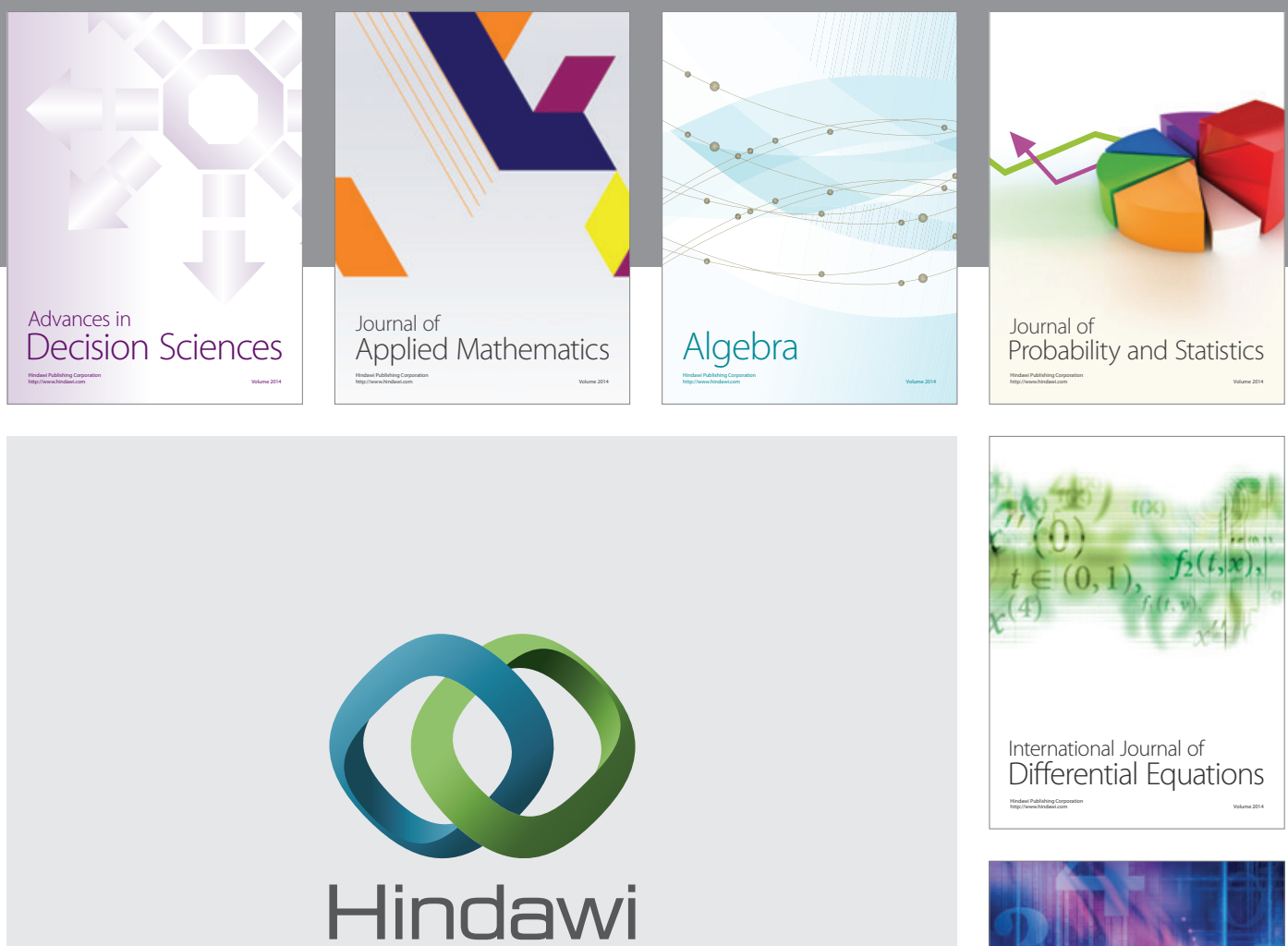

Submit your manuscripts at http://www.hindawi.com
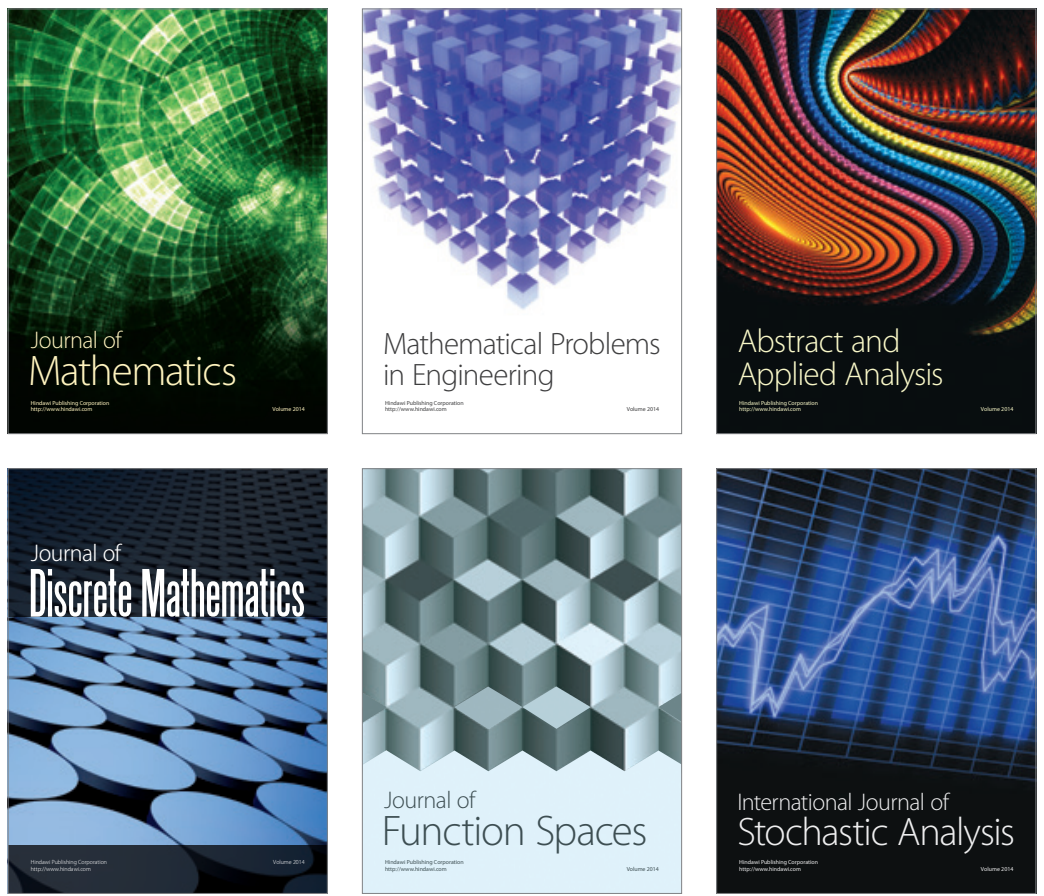

Journal of

Function Spaces

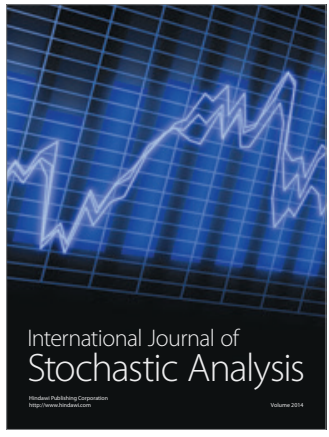

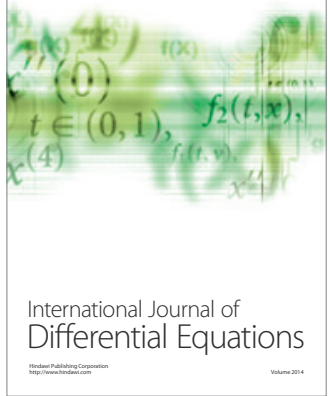
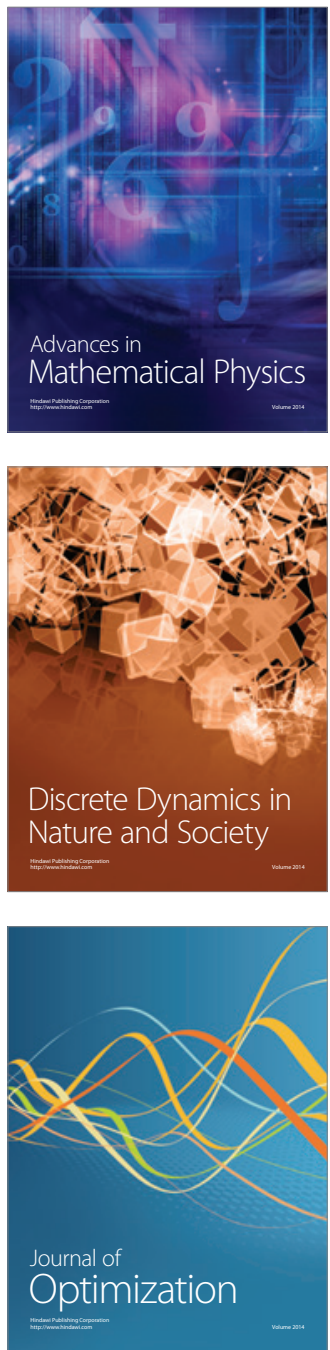